\title{
Nuevas tecnologías como estrategia terapéutica complementaria para el Trastorno de Juego
}

\author{
Susana Jiménez-Murcia ${ }^{\mathrm{abc}}$, Fernando Fernández-Aranda ${ }^{\mathrm{abc}}$, Roser Granero ${ }^{\text {bd }}$, Salomé Tárrega ${ }^{\mathrm{d}}$, \\ Neus Aymamía , Mónica Gómez-Peña a , Cristina Giner-Bartoloméa , Juan José Santamaría ${ }^{\text {, }}$ \\ Laura Forcano ${ }^{a}$, Laura Moragas ${ }^{\mathrm{a}}$, Amparo del Pino-Gutiérrez ${ }^{\mathrm{ae}}$, Marta Baño $^{\mathrm{ab}}$, José Manuel \\ Menchón ${ }^{\text {acf }}$ \\ aUnidad de Juego Patológico, Servicio de Psiquiatría, Hospital Universitario de Bellvitge-IDIBELL, Barcelona \\ ${ }^{\mathrm{b}}$ Ciber Fisiopatología Obesidad y Nutrición (CIBERObn), Instituto de Salud Carlos III \\ 'Department de Ciències Clíniques, Facultat de Medicina, Universitat de Barcelona \\ ${ }^{\mathrm{d}}$ Departament de Psicobiologia i Metodologia de les Ciències de la Salut, Universitat Autònoma de \\ Barcelona \\ eDepartament d'Infermeria de Salut Pública, Salut Mental i Maternoinfantil. Escola Universitària \\ d'Infermeria. Universitat de Barcelona.

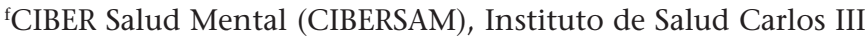

Recibido: 8-6-2015

Aceptado:3-10-2015

Nuevas tecnologías como estrategia terapéutica complementaria para el Trastorno de Juego

Resumen. El Trastorno de Juego ha sido reconocido desde hace relativamente poco tiempo. Si bien, siempre se había clasificado como un trastorno del control de los impulsos, recientemente las evidencias empíricas existentes, que demostraban la similitud con los trastornos por uso de sustancias, han favorecido que la comunidad científica acepte su categorización como adicción comportamental. Al mismo tiempo, la impulsividad y los déficits de regulación emocional, características difícilmente modificables con las terapias al uso, siguen considerándose aspectos nucleares en el desarrollo y mantenimiento del trastorno. Es por ello que las nuevas tecnologías pueden ofrecernos alternativas novedosas y eficaces para abordar estos factores asociados al Trastorno de Juego.

Palabras clave: Juego Patológico; Trastorno de Juego; impulsividad; regulación emocional; Playmancer; videojuegos terapéuticos

The use of new technology as a complementary therapeutic strategy for the treatment of gambling disorder

Summary. Problem gambling disorder has been recognized for a relatively short time. Although it had always been classified as an impulse control disorder, recently collected empirical data indicating its similarity with substance abuse disorders have driven the scientific community to accept its categorization as a behavioral addiction. At the same time, impulsiveness and emotional regulation deficit, characteristics that are difficult to modify using traditional therapy, are still viewed as being at the heart of the development and perpetuation of this disorder. Thus, new technology might offer use new and effective alternatives when confronting these factors associated with Problem Gambling.

Keywords: Problem Gambling; Gambling Disorder; impulsiveness; emotional regulation; Playmancer; therapeutic videogames

\section{Correspondencia}

Susana Jiménez-Murcia, Ph.D

Coordinadora Unidad de Juego Patológico.

Servicio de Psiquiatría.

Hospital Universitario de Bellvitge-IDIBELL y CIBERObn

c/ Feixa Llarga s/n, 08907 L'Hospitalet de Llobregat

(Barcelona).

Tel. +34 9326079 88; Fax. +34 932607658 .

E-mail: sjimenez@bellvitgehospital.cat 


\section{Introducción}

El juego ha sido una actividad de entretenimiento y ocio desde la Antigüedad. De hecho, existen evidencias de que el hombre prehistórico ya jugaba (Fontbona, 2008). A lo largo de los siglos, el juego en España ha pasado por distintas situaciones, desde la prohibición de todo tipo de juego con apuesta, hasta la legalización en 1977 (Jiménez-Murcia et al., 2014). A pesar de que, tradicionalmente, han sido las máquinas recreativas con premio las que se han asociado en mayor medida a problemas de juego en nuestro país (Becoña, 2009), en los últimos años, la proliferación de juegos en línea, de carácter global, más allá de cualquier frontera geográfica, han cambiado el perfil sociodemográfico y clínico de los pacientes que llegan a las consultas. En definitiva, en las unidades especializadas se observa cómo el Juego Patológico, denominado Trastorno de Juego en el DSM-5, está llegando a sectores de la población que, tradicionalmente, habían estado menos expuestos al riesgo de desarrollar este trastorno, por ejemplo, mujeres jóvenes o personas con elevado nivel de estudios y alto poder adquisitivo. Por ello, es posible que en los próximos años aumenten los casos con problemas de juego graves, en términos de consecuencias legales, económicas, etc. derivadas de esta actividad.

Por otra parte, a pesar de que los tratamientos psicológicos, concretamente, los de orientación cognitivoconductual y motivacionales, han demostrado ser eficaces para el control de los síntomas del Trastorno de Juego, un 30\% de pacientes presentan una pobre respuesta a este tipo de intervenciones. Los factores predictores de fracaso terapéutico se han asociado a la gravedad del cuadro, a la edad de inicio precoz, a los niveles de impulsividad subyacentes o a la psicopatología asociada. Es por ello, que desarrollar nuevas intervenciones y estrategias que puedan mejorar las que disponemos hasta ahora, es de extremo interés. En este sentido, las nuevas tecnologías pueden ofrecer una serie de ventajas y de posibilidades que conviene explorar en profundidad. En este artículo nos centraremos en describir la eficacia de los videojuegos terapéuticos o serious games para tratar trastornos relacionados con el Trastorno de Juego.

\section{Modificaciones en el DSM-5: Trastorno de Juego}

Para empezar, es interesante revisar los cambios en la clasificación diagnóstica del Juego Patológico. A pesar de que tradicionalmente, el Juego Patológico, había sido considerado un Trastorno del Control del Impulso, junto a la cleptomanía, piromanía, tricotilomanía y trastorno explosivo intermitente (DSM-IV; APA, 1994), en el DSM-5 (APA, 2013), ha pasado a denominarse Trastorno de Juego, por considerarse que «Juego Patológico» llevaba implícito un significado peyorativo (Potenza, 2013). Asimismo, con esta nueva versión, es posible especificar si el trastorno tiene un carácter episódico o persistente, si está en remisión temprana (abstinencia entre 3 y 12 meses), o mantenida (más de 12 meses). Además, es posible establecer el nivel de gravedad, considerando que el cumplimiento de 4-5 criterios sería leve, 6-7 moderado y 8-9 grave. Otro cambio significativo ha sido la reducción del punto de corte, para el establecimiento del diagnóstico, que ha pasado de 5, en el DSM-IV-TR, a 4 en el DSM-5. Esta modificación ha sido el resultado de tres estudios independientes, que han demostrado que reducir el punto de corte incrementa la sensibilidad en la identificación del trastorno y mejora la precisión diagnóstica (Stinchfield, 2005; Stinchfield et al., 2005; JiménezMurcia et al., 2009). Finalmente, el DSM-5 ha eliminado el criterio sobre actos ilegales, como parte del trastorno. Este criterio se definía como la comisión de falsificación, fraude, malversación y robo, para financiar la conducta de juego. Sin embargo, mientras que la preocupación por el juego y el deseo de recuperar las pérdidas son los síntomas más frecuentes, incluso en los casos más leves, la presencia de actos ilegales está directamente relacionada con la severidad y un peor pronóstico del trastorno (Strong y Kahler, 2007). Además, el criterio de actos ilegales no discrimina mejor la presencia del trastorno, ni la precisión diagnóstica. Estudios realizados en población general y clínica, demuestran que este criterio raramente se observa en ausencia de otros y, generalmente, se asocia a los cuadros más graves (Petry et al., 2013).

\section{Similitudes y diferencias entre el Juego Patológico y la adicción a las nuevas tecnologías}

En la Unidad de Juego Patológico, del Servicio de Psiquiatría del Hospital Universitario de Bellvitge, se realizó un estudio para comparar tres grupos de individuos, uno formado por pacientes con distintos tipos de adicciones tecnológicas, otro por jugadores patológicos tradicionales y finalmente un tercero por controles sanos. Para ello, se analizaron más de 1.000 pacientes consecutivos que habían acudido a la Unidad, entre 2005 y principios del 2009, de los cuales alrededor del $9 \%$ presentaban adicción a nuevas tecnologías. Los resultados que se obtuvieron mostraron, en primer lugar, que los distintos subtipos de adicciones tecnológicas (adicción a videojuegos en línea, otras aplicaciones de Internet y móvil) no se diferenciaban entre sí, ni en cuanto características clínicas ni tampoco en perfiles de personalidad. Asimismo, los dos grupos de pacientes (jugadores patológicos y adictos a nuevas tecnologías) presentaban, al ser comparados con los controles sanos, alteraciones marcadas en el estado de ánimo. Éste era un resultado esperable, en cuanto a que trabajos anteriores habían ya demostrado, especialmente en Juego Patológico, la elevada asociación con trastornos del estado de ánimo y de ansiedad (Kessler et al., 2008). Otro dato interesante era las similitudes en los perfiles de personalidad, entre los pacientes con Juego Patológico y los que presentaban adicciones tecnológicas. En ambos casos, este perfil se caracterizaba por elevados niveles en búsqueda de sensaciones 
y novedad, impulsividad, baja tolerancia al aburrimiento y a la monotonía, dificultades en el manejo de situaciones problemáticas, independencia, individualismo y reserva. En definitiva, estos resultados estaban en concordancia con otros estudios realizados en distintos países confirmando que, dadas las similitudes clínicas y comportamentales de estos pacientes, los programas de tratamiento podían ser muy similares (JiménezMurcia et al., 2011).

\section{Tratamiento del Juego Patológico}

La información sobre el tratamiento del Juego Patológico todavía adolece de ciertos conceptos erróneos ¿Qué se conoce realmente acerca del tratamiento del Juego Patológico en general, ya sea a juegos tradicionales o a través de internet? ¿Son eficaces? ¿Es cierto el mito de que estos trastornos no se pueden curar?

La investigación sobre resultados de tratamiento demuestra que es un trastorno que puede tratarse con éxito. Diversas publicaciones coinciden al señalar que los tratamientos psicológicos son los más eficaces, asociándose a una mejoría significativa tanto a corto como a largo plazo (Cowlishaw et al., 2012), especialmente los de orientación cognitivo-conductual, que incluyen componentes motivacionales (Rash \& Petry, 2014). Estudios realizados en la Unidad de Juego Patológico, del Hospital Universitario de Bellvitge, con amplias muestras de pacientes, que habían solicitado tratamiento profesional por su problema de juego, mostraron que la terapia cognitivo-conductual era eficaz en la mayoría de pacientes, siendo la tasa de abandonos durante el tratamiento del 30\% y la de recaídas del 23,9\% (Jiménez-Murcia et al., 2007). Al comparar el estado clínico del paciente al iniciar el tratamiento y al finalizarlo, se observaban también cambios significativos en psicopatología (alteraciones emocionales) y severidad de la conducta de juego. El riesgo de abandono y recaída decrecía significativamente tras la quinta sesión de terapia (Jiménez-Murcia et al., 2012). Es decir, parecía ser positivo un esfuerzo inicial tanto por parte del paciente, como de los familiares y también de los terapeutas. En esta misma línea, ¿podemos predecir el éxito o el fracaso de una terapia?, ¿existen datos al respecto? Los estudios científicos han demostrado que los factores asociados a una pobre respuesta al tratamiento son niveles elevados de ansiedad y depresión (en cuyo caso el juego puede actuar como una forma de aliviar estos estados) y en general gravedad de la psicopatología asociada, larga evolución de la enfermedad en el momento de iniciar tratamiento, mayor gravedad del trastorno, baja motivación al cambio y elevada impulsividad y búsqueda de sensaciones (Dunn, 2012; Jiménez-Murcia et al., 2015; Ledgerwood y Petry, 2006; Melville et al., 2007). Sin embargo, algunos rasgos de personalidad como la persistencia (autoexigencia, ambición, capacidad de esfuerzo y superación) parecen ser factores relacionados a buena respuesta al tratamiento (Jiménez-Murcia et al., 2009; Gómez-Peña et al., 2012).
Otro aspecto a tener en cuenta es que a pesar de las similitudes observadas entre los pacientes con el diagnóstico de Juego Patológico (ya sea en línea o presencial), también es cierto que existe cierta heterogeneidad en el trastorno (Álvarez-Moya et al., 2010). Así, aunque los programas de tratamiento pueden ser similares, es decir, basados en una serie de técnicas comunes en todos los casos, también es necesario evaluar y establecer las características específicas de cada paciente, tanto a nivel clínico, psicopatológico y de personalidad, como considerando el tipo de juego elegido para la adicción (estratégico versus no-estratégico) (Moragas et al., 2015). De este modo, al diseñar el plan de intervención conviene disponer de una serie de criterios para determinar la conveniencia de aplicar las distintas modalidades de intervención (individual, grupal, familiar, con tratamiento farmacológico combinado, coordinado con otros especialistas de ámbitos diversos, etc.) (Jiménez-Murcia et al., 2006).

En primer lugar, el objetivo se centrará en detener la conducta de juego, por lo que las técnicas conductuales de control de estímulos (control de dinero y evitación de situaciones de riesgo, como las conexiones a páginas de Internet, entrar en bares con máquinas o autoprohibirse la entrada de bingos y casinos) serán de utilidad en este sentido. Las técnicas cognitivas para tratar las creencias irracionales y el pensamiento mágico asociado al juego y la psicoeducación sobre las probabilidades de ganar en el juego de apuesta, serán también eficaces. El incremento de la confianza con los familiares o las personas significativas del entorno del paciente, será también un aspecto a conseguir. El entrenamiento en habilidades de afrontamiento al estrés, el aumento de la autoestima y la solución de problemas pueden ser estrategias terapéuticas necesarias para superar el trastorno. Las actividades de entretenimiento y ocio alternativas son especialmente adecuadas en un programa de deshabituación del juego.

Finalmente, hay que tener en cuenta los recursos terapéuticos necesarios para abordar los trastornos asociados al juego (abuso o dependencia de drogas o alcohol, depresión, problemas legales y financieros, etc.). De este modo, el objetivo del tratamiento, será restablecer el estilo de vida previo al trastorno y mejorar todas las áreas de la vida del sujeto afectadas o deterioradas por su enfermedad (Jiménez-Murcia et al., 2007).

En definitiva, el Juego Patológico en general y más concretamente el juego en línea es un trastorno reconocido como tal desde hace pocos años y por ello aún queda mucho por hacer, pero los avances son cada vez mayores. Ello, sin duda, se ha visto reflejado no tan sólo en los resultados de la investigación más básica, sino también a nivel clínico, como por ejemplo en el hecho de que los jugadores identifiquen y reconozcan antes el problema, con lo que cada vez es menos frecuente ver largas evoluciones de la enfermedad, presentando asimismo mayor motivación para llevar a cabo un tratamiento y por ello, mejor resultado de los programas de intervención. 


\section{Nuevas tecnologías y salud}

Cada vez más, profesionales de la salud están empezando a interesarse en enfoques terapéuticos eficientes (en términos de coste/eficacia) e innovadores. Para muchos clínicos e investigadores es de suma importancia avanzar en el tratamiento de estos trastornos, desarrollando nuevas estrategias terapéuticas, con el objetivo de no seguir aplicando las mismas intervenciones que hace tres décadas, con las limitaciones que han demostrado tener. Debido a esta razón, la utilización de las nuevas tecnologías como potenciales herramientas terapéuticas está siendo explorada por distintos grupos, (Botella et al. 2013; Claes et al., 2012; Fernández-Aranda et al., 2012; Perpiña et al., 2013). Su aplicación en el tratamiento de trastornos somáticos y psicológicos y su potencial eficacia como complementario terapéutico, está siendo motivo de interés general (Barnett et al., 2011; Coyle et al., 2005).

Las razones que han motivado la aplicación de estas nuevas estrategias terapéuticas, se basan no solo en el deseo de aprovechar todas las ventajas de las nuevas tecnologías, sino también en hacer más accesibles programas y tratamientos a los que, de otro modo, sería difícil acceder, por ejemplo en zonas rurales o en lugares donde los dispositivos asistenciales para determinadas patologías muy específicas son escasos.

Dentro de las nuevas tecnologías, los serious games, o videojuegos con fines terapéuticos, han demostrado su eficacia en diversos campos, como en el entrenamiento de habilidades y competencias específicas, manejo del dolor y modificación de emociones, conductas y actitudes (Santamaría et al., 2011). Según Griffiths (2004), las intervenciones con videojuegos, podrían tener un éxito considerable, especialmente si son diseñados específicamente para trabajar problemas concretos o habilidades específicas. En general, las razones que explicarían los aspectos beneficiosos de los videojuegos serían: 1 ) requieren altos niveles de atención y concentración, así como reacción en forma de respuestas motoras y cognitivas; 2) permiten la posibilidad de superación, es decir de adquirir una serie de habilidades y competencias que se incrementan progresivamente a medida que va aumentando la dificultad en el juego; 3) atraen a la mayoría de personas, ya que son entretenidos y motivadores; 4) facilitan la inmersión en las tareas que se están realizando y la desconexión del entorno (especialmente útiles en situaciones como en las curas de niños que han sufrido quemaduras, por ejemplo); 5) implican de forma intensiva al individuo; y 6) producen cambios en la actividad cerebral (Egerton et al., 2009; Han et al., 2011; Wilkinson et al., 2008).

En el Hospital Universitario de Bellvitge, en Barcelona, junto a otros centros de ingeniería y biotecnologías, de otros cinco países europeos, se desarrolló Playmancer (www.playmancer.eu), una iniciativa de la Unión Europea con el propósito de diseñar y aplicar un videojuego con fines terapéuticos, dirigido a intervenir en trastornos relacionados con la impulsividad, como el Trastorno de Juego. Más allá de los síntomas específicos (que ya se trataban con la terapia habitual que seguían todos los pacientes), se pretendió intervenir en los aspectos subyacentes al trastorno y que son menos susceptibles al cambio, siguiendo tratamientos tradicionales. Estos aspectos a los que nos referimos son, la impulsividad, déficits de regulación emocional, baja tolerancia a la frustración, escaso autocontrol, dificultad en la planificación de tareas y objetivos, baja solución de problemas, etc. (Jiménez-Murcia et al., 2009; Fernández-Aranda et al., 2012)

Como todo serious game, el videojuego iba más allá de lo puramente lúdico, aunque sin olvidar que también pretendía ser entretenido. Sin embargo, el objetivo principal era poder conseguir cambios actitudinales, conductuales y emocionales en los pacientes en los que se aplicaba. En este videojuego, además, se registraban las respuestas del paciente frente al escenario (reacciones fisiológicas que se captaban a través de cinco biosensores y respuestas emocionales que se recogían a partir de una cámara que grababa las expresiones faciales). Todas estas medidas interactuaban con las distintas tareas que propiciaba el videojuego. Éste se desarrollaba en una isla de un archipiélago (de ahí que el nombre del videojuego fuera Islands), en el que el sujeto debía progresar y avanzar, en base al logro de una serie de objetivos terapéuticos. Concretamente, se trataba de un videojuego de aventuras-simulación, en un escenario en el que el avatar se enfrentaba a distintos retos y/o situaciones, a través de los que podía entrenar las habilidades y/o actitudes que se pretendían incrementar (por ejemplo, resolución de problemas, control de reacciones impulsivas, afrontamiento de distintas situaciones asociadas a frustración y manejo de emociones negativas). El paciente era confrontado con distintas situaciones, más o menos complejas, en función de su estado emocional, que se evaluaba de forma continua a través de biosensores y de su expresión facial. En el videojuego, solo se superaba el nivel, es decir, se progresaba, si el estado emocional del sujeto era de calma, relajación, control y planificación. Así, el objetivo final no era ganar, en el sentido clásico de los juegos, sino conseguir una mayor capacidad de autocontrol. En todo momento, el paciente recibía feedback sobre sus logros.

Más concretamente, el videojuego constaba de tres de mini-juegos, que se describen a continuación:

La Cara de Cronos: el paciente necesitaba planificar un recorrido para poder escalar un arrecife, evitando obstáculos producidos por sus emociones negativas como piedras o pájaros, que le podían hacer caer. Con este mini-juego se pretendía entrenar al paciente en capacidad de planificación, aumento de la tolerancia a la frustración, entrenamiento en demora de respuesta y mejora del auto-control.

Los Tesoros del Mar: el paciente buceaba, exploraba el fondo del océano, conseguía peces y tesoros marinos, mientras controlaba su gasto de oxígeno. La dificultad de la tarea se asociaba, de nuevo, a su estado emocional. La frustración, impulsividad y rapidez en 


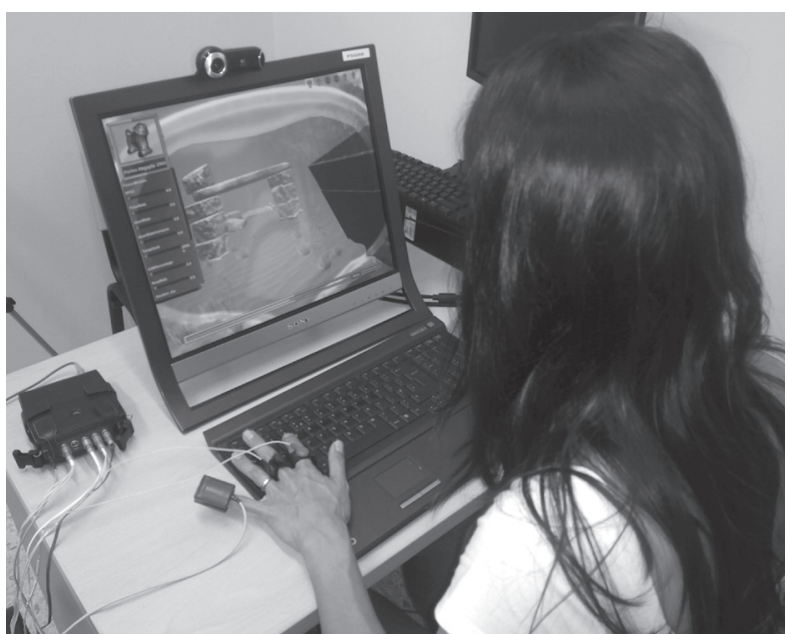

Figura 1. Registro de la actividad fisiológica a través de Playmancer.

las tareas, complicaba y disminuía los logros en el mini-juego. Los objetivos eran el entrenamiento en solución de problemas, el auto-control y la regulación emocional.

Los Signos de Magupta: consistía en un mini-juego de relajación, en el que el paciente podía unir las estrellas de cinco constelaciones estelares, a través de respiraciones lentas y profundas. Se trataba del espacio de la isla que el sujeto debía visitar, cuando se encontraba en un estado de tensión, estrés y alteración. De hecho, cuando el propio videojuego (a través de los biosensores y la cámara que registraba la expresión facial del paciente) identificaba alguna emoción negativa, indicaba al paciente que debía realizar la tarea de relajación. Una vez conseguido este estado, el sujeto podía seguir jugando en el mismo punto en el que lo había dejado. El objetivo de esta tarea era el entrenamiento en relajación, el aumento de auto-conciencia y la auto-regulación.

Respecto al procedimiento, el videojuego se aplicaba de forma complementaria a la terapia estándar, a los pacientes que acudían a tratamiento por su problema de Trastorno de Juego. Con el objetivo de controlar posibles efectos adversos del videojuego, éste solo se podía utilizar en el contexto hospitalario, siendo todas las sesiones supervisadas por un terapeuta. Cada sesión consistía en 20 minutos de juego. Antes y después de la exposición a los mini-juegos del serious game, el sujeto realizaba 3 minutos de relajación en uno de los escenarios de la isla, mientras oía una música relajante, sonidos de aves, etc. La frecuencia de las sesiones era semanal, realizándose un total de 10 , coincidiendo con las visitas para la terapia estándar.

Hasta el momento, este videojuego sólo se ha utilizado en un contexto hospitalario dado que, inicialmente, era necesario analizar su eficacia como terapia complementaria al tratamiento habitual indicado en esta patología. Asimismo, toda la tecnología y dispositivos que requería este procedimiento complicaban su uso, fuera de este entorno. De hecho, la plataforma del videojuego integraba distintos dispositivos como cinco biosensores (reacción galvánica de la piel, temperatura, frecuencia respiratoria, tasa cardíaca y saturación de oxígeno), medidos a través de un sistema basado en el MobiHealth MobileTM@ y una cámara, que grababa las expresiones faciales del paciente, extrayendo algoritmos que permitían el reconocimiento de emociones (ira, diversión y aburrimiento). Como se ha mencionado anteriormente, estos dispositivos detectaban el estado emocional y las reacciones fisiológicas del sujeto de forma continua, durante toda la sesión de juego, mientras era confrontado a tareas específicas, que podían desencadenar tensión o estrés (dificultad para avanzar, conseguir objetos, etc.). En el momento en el que la plataforma detectaba un estado emocional negativo o reacciones fisiológicas sugestivas de un estado de estrés, el videojuego dirigía al avatar (paciente) a la zona de relajación hasta que éste se relajaba.

Tomando en consideración el feedback de los pacientes, la mayoría refería una experiencia positiva después de la utilización de esta plataforma. De hecho, podríamos resumir los resultados en los siguientes puntos: 1) los pacientes con diagnóstico de Trastorno de Juego se sentían cómodos utilizando el videojuego (usabilidad entorno al 85\%); 2) el videojuego era capaz de provocar respuestas emocionales y reacciones fisiológicas en los sujetos (al comparar éstas con las presentadas por sujetos control, los pacientes mostraban una sobre-reacción ante las distintas tareas); 3) era posible identificar emociones positivas y negativas asociadas a determinadas reacciones fisiológicas; y 4) del mismo modo que en otros estudios, el entrenamiento en relajación y el biofeedback continuo reducían significativamente la tensión y el estrés producido por distintas situaciones del videojuego.

Asimismo, uno de los estudios realizados para validar la plataforma Playmancer, que tuvo como objetivos específicos evaluar los cambios pre-post tratamiento en las medidas de impulsividad, expresión de la ira y psicopatología general, en un grupo de 16 pacientes con diagnóstico de Trastorno de Juego, grave (según criterios DSM-5), informó de resultados positivos, que se detallan a continuación.

Todos los pacientes recibieron 16 sesiones de terapia grupal, de orientación cognitivo-conductual, dirigida a los síntomas específicos del Trastorno de Juego. Las técnicas utilizadas fueron: entrevista motivacional, control de estímulos (control de dinero, evitación situaciones de riesgo y autoprohibición de juego presencial y en línea, dependiendo del tipo de juego problemático de cada paciente), técnicas cognitivas (entrenamiento auto-instruccional, solución de problemas y reestructuración cognitiva), refuerzo y autorrefuerzo, entrenamiento en habilidades y prevención de recaídas. Asimismo, de forma complementaria, estos pacientes recibían 20 minutos de práctica con Playmancer, a lo largo de 10 sesiones adicionales. Los resultados permiten apreciar cambios significativos en las medidas de impulsividad, expresión de la ira y psicopatología general. El riesgo de abandono y recaída, durante el tratamiento, se situaba en el $12.5 \%$ para 
abandono y $37.5 \%$ para recaída. Sin embargo, lo más significativo era que, teniendo en cuenta que se trataba de un grupo de pacientes con un problema de juego grave (más del 40\% habían cometido actos delictivos, criterio establecido para evaluar severidad del trastorno), se observaba un cambio muy significativo en las medidas de impulsividad y psicopatología. En los estudios de tratamiento, habitualmente, éste es el perfil de paciente que presenta una pobre respuesta a la terapia cognitivo-conductual, y sin embargo en este caso los resultados eran más que satisfactorios (incluso la tasa de abandonos era significativamente menor a la habitualmente observada en investigaciones previas). La principal conclusión del estudio es que complementar el tratamiento psicológico usual con una estrategia terapéutica específica, basada en los serious games, puede ser útil para abordar aquellas características y rasgos de base que presentan los pacientes con diagnóstico de Trastorno de Juego, como pueden ser la elevada impulsividad o los déficits en regulación emocional.

Agradecimientos:

Este proyecto de investigación ha contado con la financiación de la Unión Europea (Séptimo Proyecto Marco, I+D/ FP7-ICT-215839-2007), Fondo de Investigación Sanitaria (FIS/ PI081573), PSI2014-56303-REDT: PROMOSAM y del CIBEROBN (Fisopatología de la Obesidad y Nutrición; CB06/03) del Instituto Carlos III. Agradecer a los miembros de Playmancer Consortium su participación en este proyecto.

\section{Referencias}

Álvarez-Moya, E., Jiménez-Murcia, S., Aymamí, M. N., Gómez-Peña, M., Granero, R., Santamaría, J. J., Menchon, J. M., \& Fernández-Aranda, F. (2010). Subtyping study of a male pathological gambling sample. Canadian Journal of Psychiatry, 55(8), 498-506.

American Psychiatric Association, APA. (2000). Diagnostic and statistical manual of mental disorders (4th ed. Revised). Washington, DC: American Psychiatric Association.

American Psychiatric Association, APA. (2013). Diagnostic and statistical manual of mental disorders (5th ed.). Washington, DC: American Psychiatric Association.

Barnett, A., Cerin, E., \& Baranowski, T. (2011). Active video games for youth: a systematic review. Journal of Physical Activity \& Health, 8(5), 724-737.

Becoña, E. (2009). Spain. En G. Meyer, T. Hayer \& M. Griffiths (Eds.), Problem gambling in Europe. Challenges, prevention, and interventions (pp. 281-298). Nueva York: Springer.

Botella, C., Bretón-López, J., Quero, S., Baños, R., \& García-Palacios, A. (2010). Treating cockroach phobia with augmented reality. Behavior Therapy, 41(3), 401-413.

Claes, L., Jiménez-Murcia, S., Santamaría, J. J., Moussa, M. B., Sánchez, I., Forcano, L., Magnenat-Thalmann, N., Konstantas, D., Overby, M. L., Nielsen, J., Bults,
R. G., Granero, R., Lam, T., Kalapanidas, E., Treasure, J., \& Fernández-Aranda, F. (2012). The facial and subjective emotional reaction in response to a video game designed to train emotional regulation (Playmancer). European Eating Disorders Review, 20(6), 484-9. doi:10.1002/erv.2212.

Cowlishaw, S., Merkouris, S., Dowling, N., Anderson, C., Jackson. A., \& Thomas, S. (2012). Psychological therapies for pathological and problem gambling (Review). The Cochrane Library. doi: 10.1002/14651858. CD008937.pub2.

Coyle, D., Matthews, M., Sharry, J., Nisbet, A., \& Doherty, G. (2005). Personal investigator: A therapeutic 3D game for adolescent psychotherapy. Journal of Interactive Technology \& Smart Education, 2(2), 73-88.

Dirección General de Ordenación del Juego. Memoria Anual, 2011. Madrid: Ministerio de Hacienda y Administraciones Públicas.

Dunn, K., Delfabbro, P., \& Harvey, P. (2012). A Preliminary, Qualitative Exploration of the Influences: Associated with Drop-Out from Cognitive-Behavioural Therapy for Problem Gambling: An Australian Perspective. Journal of Gambling Studies, 28, 253-272. doi 10.1007/s10899-011-9257-X.

Egerton, A., Mehta, M. A., Montgomery, A. J., Lappin, J. M., Howes, O. D., Reeves, S. J., Cunningham, V. J., \& Grasby, M. (2009). The dopaminergic basis of human behaviors: A review of molecular imaging studies. Neuroscience and Biobehavioral Reviews, 33(7), 1109-1132.

Fontbona, M. (2008). Historia del Juego en España: de la Hispania Romana a nuestros días. Barcelona: Flor del Viento.

Granero, R., Penelo, E., Martínez-Giménez, R., AlvarezMoya, E., Gómez-Peña, M., Aymamí, M. N., Bueno, B., Fernández-Aranda, F., \& Jiménez-Murcia, S. (2009). Sex differences among treatment-seeking adult pathologic gamblers. Comprehensive Psychiatry, 50(2), 173-180. doi:1016/j.comppsych.2008.07.005.

Granero, R., Penelo, E., Stinchfield, R., FernándezAranda, F., Savvidou, L. G., Fröberg, F., Aymamí, N., Gómez-Peña, M., Pérez-Serrano, M., Del Pino-Gutiérrez, A., Menchón, J. M., \& Jiménez-Murcia, S. (2013). Is Pathological Gambling Moderated by Age? Journal of Gambling Studies, 30(2); 475-492. doi:10.1007/ s10899-013-9369-6.

Griffiths, M. (1999). Gambling technologies: Prospects for problem gambling. Journal of Gambling Studies, $15,265-283$.

Griffiths, M. (2004). Betting your life on it: Problem gambling has clear health related consequences. British Medical Journal, 329(7474), 1055-1056.

Griffiths, M., Wood, R., Parke, J. \& Parke, A. (2007). Gaming research and best practice: Gaming industry, social responsibility and academia. Casino and $G a-$ ming International, 3, 97-103.

Griffiths, M., Wood, R., \& Parke, J. (2008). Reducing addiction risk in developing online games. World Online Gambling Law Report, 7, 15-16.

Han, D. H., Bolo, N., Daniels, M. A., Arenella, L., Lyoo, 
I. K., \& Renshaw, P. F. (2011). Brain activity and desire for internet video game play. Comprehensive Psychiatry, 52(1), 88-95.

Jiménez-Murcia, S., Aymamí, M. N, Gómez-Peña, M., Álvarez-Moya, E. M, \& Vallejo, J. (2006). Protocols de tractament cognitivoconductual pel joc patològic $i$ d'altres addicions no tòxiques. Barcelona: Hospital Universitari de Bellvitge, Departament de Salut, Generalitat de Catalunya.

Jiménez-Murcia, S., Álvarez-Moya, E. M., Aymamí, M. N., Gómez-Peña, M., Jaurrieta, N., Sans, B., RodríguezMartí, J. \& Vallejo, J. (2007). Cognitive-behavioral group treatment for pathological gambling: Analysis of effectiveness and predictors of therapy outcome. Psychotherapy Research, 17(5), 544-552.

Jiménez-Murcia, S., Aymamí, N., Gómez-Peña, M., Santamaría, J. J, Álvarez-Moya, E., Fernández-Aranda, F., Granero, R., Penelo, E., Bueno, B., Moragas, L., Gunnard, K., \& Menchón, J. M. (2012). Does exposure and response prevention improve the results of group cognitive-behavioural therapy for male slot machine pathological gamblers? British Journal of Clinical Psychology, 51(1), 54-71. doi:10.1111/j.20448260.2011.02012.x.

Jiménez-Murcia, S., Fernández-Aranda, F., Granero, R., \& Menchón, J. M. (2014). Gambling in Spain: update on experience, research and policy. Addiction, 109, 1595-1601. doi: 10.1111/add.12232.

Jiménez-Murcia, S., Stinchfield, R., Álvarez-Moya, E., Jaurrieta, N., Bueno, B., Granero, R., Aymamí, N. M., Gómez-Peña, M., Gímenez-Martínez, R., \& Fernández-Aranda, F. (2009). Reliability, validity, and classification accuracy of a Spanish translation of a measure of DSM-IV diagnostic criteria for pathological gambling. Journal of Gambling Studies, 25(1), 93-104.

Jiménez-Murcia, S., Álvarez-Moya, E., Stinchfield, R., Fernández-Aranda, F., Granero, R., Aymamí, N., Gómez-Peña M., Jaurrieta N., Bove F.\& Menchón, J. M. (2010). Age of onset in pathological gambling: clinical, therapeutic and personality correlates. Journal of Gambling Studies, 26(2), 235-248. doi: 10.1007/ s10899-009-9175-3.

Jiménez-Murcia, S., Fernández-Aranda, F., Santamaría, J. J., Granero, R., Penelo, E., Gómez-Peña, M., Aymamí, M. N., Moragas, L., Soto, A. \& Menchón, J. M (2011). A comparative study between technological addictions and pathological gambling: more similarities than differences? International Gambling Studies, 11(3), 325-337.

Jiménez-Murcia S., Granero R., Aymamí N., GómezPeña, M., Moragas L., del Pino-Gutiérrez, A., Santamaría J. J., Menchón J. M. \& Fernández-Aranda F. (2013) Nuevas Tecnologías: desde la adicción hasta la rehabilitación. En Laespada T. \& Estevez A. (Eds.) ¿Existen las adicciones sin sustancias? (pp. 101-116) Bilbao: Instituto Deusto de Drogodependencias. Recuperado de https://download.e-bookshelf.de/ download/0002/2881/79/L-G-0002288179-000312 9221.pdf.
Jiménez-Murcia, S., Granero, R., Fernández-Aranda, F., Arcelus, J., Aymamí, M. N, Gómez-Peña, M., Tárrega, S., Moragas, L., Del Pino-Gutiérrez, A., Sauchelli, S., Fagundo, A. B., Brewin, N., \& Menchón, J. M. (2015). Predictors of Outcome among Pathological Gamblers Receiving Cognitive Behavioral Group Therapy. European addiction research, 21(4), 169-178. doi: 10.1159/000369528.

Kessler, R. C, Hwang, I., LaBrie, R., Petukhova, M., Sampson, N. A., Winters, K. C., \& Shaffer, H. J. (2008). DSM-IV pathological gambling in the National Comorbidity Survey Replication. Psychological medicine, 38, 1351-1360. doi:1017/S0033291708002900.

Ledgerwood, D. M., \& Petry, N. M. (2006). What do we know about relapse in pathological gambling? Clinical Psychology Review, 26(2), 216-228.

Moragas, L., Granero, R., Stinchfield, R., FernándezAranda, F., Fröberg, F., Aymamí, N., Gómez-Peña, M., Fagundo, A. B., Islam, M. A., Del Pino-Gutiérrez, A., Agüera, Z., Savvidou, L. G., Arcelus, J., Witcomb, G. L., Sauchelli, S., Menchón, J. M., \& Jiménez-Murcia, S. (2015). Comparative analysis of distinct phenotypes in gambling disorder based on gambling preferences. BMC Psychiatry, 15, 86. doi: 10.1186/s12888015-0459-0.

Melville, K. M., Casey, L. M., \& Kavanagh, D. J. (2007). Psychological treatment dropout among pathological gamblers. Clinical Psychology Review, 27(8), 944-958. Odlaug, B. L., Marsh, P. J., Kim, S. W., \& Grant, J. E. (2011). Strategic vs nonstrategic gambling: characteristics of pathological gamblers based on gambling preference. Annals of clinical psychiatry, 23(2), 105-112.

Perpiñá, C., Roncero, M., Fernández-Aranda, F., Jiménez-Murcia, S., Forcano, L., \& Sánchez, I. (2013). Clinical validation of a virtual environment for normalizing eating patterns in eating disorders. Comprehensive psychiatry, 54(6), 680-686. doi: 10.1016/j.comppsych.20.

Petry, N. M., Blanco, C., Auriacombe, M., Borges. G., Bucholz, K., Crowley, T. J., Grant, B.F., Hasin, D. S, \& O'Brien, C. (2013). An Overview of and Rationale for Changes Proposed for Pathological Gambling in DSM-5. Journal of Gambling Studies, 30(2), 493-502.

Rash C. J., Petry N. M. (2014). Psychological treatments for gambling disorder. Psychology research and behavior management, 7, 285-295. doi: 10.2147/PRBM.S40883.

Santamaría, J. J., Soto, A., Fernández-Aranda, F., Krug, I., Forcano, L., Gunnard, K., Kalapanidas, E., Lam, T., Raguin, T., Davarakis, C., Menchón, J. M., \& JiménezMurcia, S. (2011). Serious games as additional psychological support: A review of the literature. Journal of CyberTherapy \& Rehabilitation , 4(4).

Stinchfield, R. (2003). Reliability, validity, and classification accuracy of a measure of DSM-IV diagnostic criteria for pathological gambling. American Journal of Psychiatry, 160(1), 180-182.

Stinchfield, R., Govoni, R., \& Frisch, G. R. (2005). DSMIV diagnostic criteria for pathological gambling: Reliability, validity, and classification accuracy. American Journal on Addictions, 14(1), 73-82. 
Stinchfield, R., Kushner, M. G., \& Winters, K. C. (2005). Alcohol use and prior substance abuse treatment in relation to gambling problem severity and gambling treatment outcome. Journal of Gambling Studies, 21(3), 273-297.

Strong, D. R., \& Kahler, C. W. (2007). Evaluation of the continuum of gambling problems using the DSM-IV. Addiction, 102(5), 713-721.

Wilkinson, N., Ang, R.P., \& Goh, D.H. (2008). Online video game therapy for mental health concerns: A review. International Journal of Social Psychiatry, 54(4), 370-382.

\section{Noves tecnologies com a estratègia terapèutica complementària per al Trastorn de Joc}

Resum. El Trastorn de Joc ha estat reconegut des de fa relativament poc temps. Si bé, sempre s'havia classificat com un trastorn del control dels impulsos, recentment les evidències empíriques existents, que demostraven la similitud amb els trastorns per ús de substàncies, han afavorit que la comunitat científica accepti la seva categorització com a addicció comportamental. Alhora, la impulsivitat i els dèficits de regulació emocional, característiques difícilment modificables amb les teràpies a l'ús, segueixen considerant aspectes nuclears en el desenvolupament i manteniment del trastorn. És per això que les noves tecnologies poden oferir-nos alternatives innovadores $i$ eficaces per abordar aquests factors associats al Trastorn de Joc.

Paraules clau: Joc Patològic; Trastorn de Joc; impulsivitat; regulació emocional; Playmancer; videojocs terapèutics 